\title{
Crecimiento y crisis en la región metropolitana de Madrid: significado y contradicciones de la economía del conocimiento ${ }^{1}$
}

\author{
Ricardo Méndez Gutiérrez del Valle²
}

\begin{abstract}
RESUMEN
La región metropolitana de Madrid ha vivido en las dos últimas décadas importantes transformaciones que la consolidaron como principal centro económico con funciones de comando de la Europa meridional, con un rápido aumento de sus indicadores de producción y consumo, su volumen de población inmigrante y la superficie ocupada, al generalizarse los procesos de suburbanización de baja densidad. Pero esa tendencia se ha visto acompañada por importantes costes sociales y ambientales, una intensa crisis desde 2008 y la profundización de las desigualdades socioespaciales en su interior. La progresiva expansión de la economía del conocimiento resulta un buen exponente de este tipo de procesos esencialmente contradictorios que marcan la integración de las megaciudades en el capitalismo global y las nuevas formas territoriales asociadas.
\end{abstract}

Palabras clave: Región metropolitana, globalización, economía del conocimiento, mercados de trabajo, Madrid.

\begin{abstract}
The metropolitan region of Madrid has undergone significant changes in the last two decades, establishing it as the main economic centre with command functions in Southern Europe. This has resulted in a rapid increase in its rates of production and consumption, the size of its immigrant population and the processes of lowdensity suburbanisation (sprawl) becoming more common. But this trend, which can be linked to its prominent position in the world cities network, has been accompanied by significant social and environmental costs, an abrupt crisis since 2008 and the deepening of socio-spatial inequalities within. The progressive growth of the knowledge economy is a good indicator of this type of essentially contradictory process which signals the integration of megacities into global capitalism and the associated territorial changes.
\end{abstract}

Key words: Metropolitan region, globalization, knowledge economy, labour markets, Madrid.

1. El artículo se integra dentro del proyecto de investigación financiado por el Plan Nacional de I+D+i del Ministerio de Ciencia e Innovación de España sobre las regiones metropolitanas españolas en la sociedad del conocimiento: tendencias económicas y transformaciones territoriales (CSO200910888). Artículo recibido el 31 de agosto de 2011, aceptado el 9 de enero de 2012 y corregido el 6 de febrero de 2012.
2 Instituto de Economía, Geografía y Demografía, Centro de Ciencias Humanas y Sociales del Consejo Superior de Investigaciones Científicas (España).

E-mail: ricardo.mendez@cchs.csic.es 
Desde el inicio del proceso de globalización y, de modo especial, en el transcurso de la última década, Madrid se ha consolidado como metrópoli de primer nivel dentro de la Unión Europea. Tanto la recuperación del dinamismo demográfico asociado a la inmigración exterior, como un rápido crecimiento económico que incrementó el volumen de ocupados y el nivel medio de ingresos de sus ciudadanos, fueron algunos de los indicadores más utilizados para atestiguarlo. Al mismo tiempo, la concentración de sedes de grandes empresas y de servicios avanzados la situaron en una posición destacada dentro de la red de ciudades mundiales, mejorando también la valoración de su capacidad para localizar inversiones, junto a su ambiente cultural y los niveles de bienestar de la mayoría de sus ciudadanos.

Ese proceso se acompañó por otro de expansión superficial hasta alcanzar dimensiones propias de una verdadera región metropolitana. Frente al modelo de ciudad compacta característico de la Europa mediterránea, se generalizó el desarrollo de espacios residenciales de baja densidad a lo largo de una red de transportes que se densificó para convertirse en aliada indispensable de las estrategias inmobiliarias. La difusión de numerosas empresas, relocalizadas en nuevas áreas de actividad situadas en ciudades metropolitanas cada vez más alejadas de la capital, transformó un territorio tradicionalmente mononuclear en otro policéntrico, al tiempo que la urbanización desbordaba los límites administrativos de la Comunidad Autónoma de Madrid para alcanzar a las regiones limítrofes.

Pero esa metamorfosis, asociada para algunos a su exitosa inserción en la globalización capitalista, ha estado también marcada por profundas contradicciones que deben entenderse como contrapunto inherente a la lógica del sistema, favorecidas por una coalición de actores locales que dio prioridad a la lógica del crecimiento y convirtió el suelo en la mercancía de más alta rentabilidad, solo comparable a la derivada de la especulación financiera. Apenas en unas pocas ciudades metropolitanas se planteó una orientación del planeamiento urbano y las políticas de desarrollo preocupada por atenuar algunos de los costes sociales y ambientales derivados, sin que un gobierno regional de inspiración neoliberal llegase a definir una estrategia territorial para el conjunto.

Tras más de un decenio de explosión urbana sin precedentes, Madrid se ha sumido en una profunda crisis desde finales de 2007, que no solo ha frenado de forma brusca las optimistas previsiones anteriores sino que cuestiona su modelo de crecimiento. Este también se basó en la expansión del capital financiero, la masiva construcción de viviendas asociada a la llamada burbuja inmobiliaria y la proliferación de servicios al consumo de baja productividad, que contrastó con un intenso proceso desindustrializador, una pérdida de competitividad exterior y el aumento de la precariedad laboral. En el plano territorial, la progresiva eliminación de restricciones a la localización propició una redistribución de las empresas y los grupos sociolaborales que alteró -pero no eliminólas divisorias heredadas entre áreas centrales y periféricas o entre sectores de la aglomeración, en un contexto general de creciente segmentación y nacimiento de nuevas formas de desigualdad socioespacial.

Con esa trama argumental, el objetivo del artículo es doble: por una parte, describir e interpretar su evolución económica reciente, junto a algunas contradicciones de un modelo de crecimiento generador de elevados costes e importantes cambios en la estructura metropolitana, que parece agotado tras el inicio de la actual crisis; al mismo tiempo, ahondar en el significado de la llamada economía del conocimiento, convertida en objetivo central de numerosos discursos regeneradores en la Unión Europea desde la Ilamada Estrategia de Lisboa (2000). Se pretende mostrar su importancia actual, la estricta lógica que preside las estrategias de localización de actividades desmaterializadas en bastantes casos, junto a las nuevas formas de desigualdad territorial que introduce, solo visibles cuando se incorporan visiones transversales superadoras de la clásica división sectorial de la actividad económica.

De este modo, tras un breve apartado teórico que define el contexto de la investigación, los dos siguientes epígrafes resumen algunas de las transformaciones demográficas, económicas y territoriales que mejor identi- 
fican lo ocurrido en Madrid $^{3}$ en las últimas décadas, apuntando claves interpretativas. En los dos últimos, el foco de atención se dirige hacia la economía del conocimiento, analizada desde una doble perspectiva: en primer lugar, a partir de la importancia y distribución de aquellas actividades que se consideran más intensivas en el uso del conocimiento; en segundo lugar, a partir del estudio de los contratos a profesionales altamente cualificados en los cinco últimos años, considerando tanto sus estrictas pautas de distribución como la diversa estabilidad del empleo generado. Eso permite revisar de forma crítica algunas afirmaciones habituales en una parte de la investigación reciente, derivando algunas conclusiones a contrastar con las obtenidas en otras áreas urbanas.

El artículo propone, por tanto, un enfoque que puede calificarse de neoestructuralista en cuanto centra su atención en el impacto de las tendencias inherentes a la actual fase de desarrollo capitalista sobre la nueva configuración económica y territorial metropolitana, valorando el significado de un conjunto de actividades y profesionales que suscitan creciente atención. No obstante, se comparte la idea de que, para profundizar en las claves explicativas de las diferentes respuestas dadas por las metrópolis a estos procesos y su diversa manifestación espacial es preciso "realizar una lectura de la forma en que operan y se organizan los agentes sociales en la esfera local -la más inmediata-, pues son sus acciones (o inacciones) y los procesos sociales asociados los que sostienen la construcción de la realidad territorial" (Hidalgo y Zunino, 2011: 81), lo que supondrá prestar mayor atención al entorno institucional y aplicar metodologías con mayor componente cualitativo, ajenas a los objetivos y posibilidades de este texto.
3 Aunque existen otras delimitaciones de carácter funcional, aquí se identifica la región metropolitana de Madrid con el territorio de la Comunidad Autónoma, de unos ocho mil kilómetros cuadrados, tal como se hace en la mayoría de investigaciones, al contar así con información para abordar los objetivos planteados.

\section{Las regiones metropolitanas en el capitalismo global: tendencias económicas y ajustes territoriales}

En el marco que define la globalización capitalista, las grandes aglomeraciones metropolitanas constituyen observatorios privilegiados para analizar e interpretar las tendencias y contradicciones asociadas a esta fase en la evolución del sistema, así como las respuestas locales específicas relacionadas con la trayectoria histórica, los recursos, los actores y las instituciones presentes en cada caso. Desde obras como las de Soja (2000) sobre la postmetrópolis, o de Scott (2003) sobre la ciudad-región global, en la última década se han sucedido diversas propuestas generalizadoras para comprender estos procesos, apoyadas a su vez en un elevado número de estudios monográficos. Sin pretender en ningún caso una revisión pormenorizada de esa literatura, pueden recordarse y, en su caso, matizar aquellos planteamientos sobre la nueva geografía económica metropolitana que guardan relación directa con la investigación realizada.

En relación con los cambios en su base económica, tres son las tendencias más repetidas con carácter general. La primera y más visible es su terciarización, que reduce la participación relativa de unas actividades productivas enfrentadas a crecientes deseconomías y movimientos de deslocalización selectiva; crecen, en cambio, tanto aquellos servicios especializados a las empresas que ocupan el suelo de forma más intensiva y rentable y contribuyen al impulso de la innovación (Simmie y Strambach, 2006), como aquellos otros relacionados con la expansión de múltiples formas de consumo masivo, a los que algunos autores han otorgado un protagonismo poco habitual en los estudios precedentes (Glaeser et al., 2001). Tendencia complementaria es la que convierte a estas grandes aglomeraciones en motores de la desmaterialización del sistema productivo (Halbert, 2005), lo que no solo supone una creciente especialización en servicios pertenecientes a la esfera de la intermediación -que gestionan los flujos materiales e inmateriales que articulan el sistema-, o a la esfera 
de la reproducción social -que incluye todo tipo de servicios a la población y administrativos-, sino también que buena parte de las empresas industriales que permanecen tan solo localizan aquí funciones ajenas a las de fabricación, progresivamente desplazadas. El tercer rasgo que parece afirmarse, aunque de modo desigual, es la concentración en estas áreas de lo que se define como economía del conocimiento, constituida tanto por actividades industriales como de servicios intensivas en la producción y uso de este recurso estratégico, asociadas a un mayor esfuerzo de innovación y que exigen unos recursos humanos, así como un entorno territorial e institucional con características específicas, lo que limita los riesgos de deslocalización y se convierte en criterio esencial para identificar ahora las nuevas jerarquías interurbanas e intrametropolitanas (Krätke, 2007; Winden, Berg \& Pol, 2007). De ahí la progresiva atención prestada a la consolidación de instituciones, redes y políticas urbanas capaces de favorecer su desarrollo y una mejor concertación entre los diferentes actores del sistema de innovación regional y local, avanzando así hacia una gobernanza relacional.

Desde la perspectiva del empleo, la idea más repetida ha sido aquella que considera la progresiva dualización de su mercado de trabajo, afirmación que en ningún caso pretende reducir la complejidad laboral metropolitana a una realidad dicotómica, sino destacar que se refuerza la presencia de dos tipos de trabajadores situados en los extremos de ese continuo. Un primer grupo corresponde a los trabajadores altamente cualificados, que la teoría del capital humano siempre consideró un activo específico movilizable para aumentar la eficiencia productiva e impulsar la competitividad regional y urbana (Romer, 1989) y que ahora ve revalorizada su importancia en propuestas como las de Stehr (2002) sobre los trabajadores del conocimiento, o la de Florida (2002) sobre la clase creativa, asociados a mejores condiciones laborales y salariales. Su contrapunto es la proliferación de lo que Gorz (1991) calificó como los nuevos servidores, inmersos en un mercado secundario de trabajo en donde la precariedad, los bajos ingresos, las malas condiciones de trabajo, las dificultades de promoción y, en ciertos casos, la informalidad son rasgos definitorios (Castillo, 2005). Aunque presentes en todo tipo de actividades, es en la construcción, el comercio minorista y multitud de servicios a la población donde cobran mayor importancia, así como entre la población inmigrante y otros grupos de riesgo.

Consecuente con estas transformaciones, se asiste a una recomposición de la forma metropolitana para la que existe amplia coincidencia en buena parte de los diagnósticos realizados en estos años (Dematteis, 1998; Méndez, 2007; De Mattos, 2010). Se afirma así una metropolización expandida, en la que los procesos de difusión residencial de baja densidad se complementan con el traslado de un número creciente de empresas y empleos, que tienden a condensarse en ciertos subcentros polarizadores de un creciente volumen de flujos diarios, lo que favorece la conformación de estructuras policéntricas y áreas urbanas de dimensión regional. Las estrategias empresariales de segmentación productiva, la mejora de los transportes y la revolución de las comunicaciones, la promoción inmobiliaria y de áreas empresariales en las periferias metropolitanas, junto a políticas de inversión pública en infraestructuras, o la desregulación del mercado de suelo, convergen con desigual importancia según los casos para propiciar esa postsuburbia compleja, dinámica y sometida también a fuertes tensiones por la multiplicidad de actores e intereses que en ella se confrontan (Borsdorf, 2005). En ausencia de un planeamiento urbanístico de ámbito metropolitano con capacidad reguladora, tales procesos también favorecen una creciente segmentación socioespacial. Complemento de esta tendencia es la ruptura de la continuidad como elemento definidor de lo urbano, al aparecer áreas dispersas y en ocasiones muy alejadas del núcleo, pero funcionalmente integradas con él, lo que cuestionaría el significado actual de la distancia física en un territorio progresivamente aplanado y hace inútil cualquier intento de dibujar los límites externos de esas aglomeraciones.

Pese al esquematismo de su presentación, este es el marco de conceptos e interpretaciones teóricas que sirve de base a la investigación que a continuación se presenta. No obstante, antes de dar paso al análisis, es preciso explicitar algunas otras ideas que pretenden revisar $\mathrm{o}$, al menos, matizar afirmaciones como las presentadas y que también se han sometido a contrastación en el caso de la aglomeración de Madrid: i) el proceso 
de globalización conllevan una creciente vulnerabilidad de los territorios por lo que, incluso aquellos que en un momento dado parecen convertirse en ejemplos de éxito en cuanto a su inserción global, están sometidos a elevados riesgos e incertidumbres ante la volatilidad actual de las ventajas competitivas acumuladas; ii) pese a su indudable importancia para promover estrategias de desarrollo económico de mayor calidad, es necesario evitar la aceptación acrítica de las narrativas sobre el conocimiento que hoy proliferan y que pueden conducir a mitificar sus efectos, a partir de una investigación más pormenorizada sobre sus características, importancia, dinamismo e impactos (económicos, sociolaborales, territoriales, ambientales, etc.), especialmente en los ámbitos metropolitanos donde se concentran este tipo de actividades y empleos; iii) los procesos espaciales dominantes y las grandes tendencias que definen la evolución de los territorios metropolitanos no pueden olvidar la multiplicidad de actores, intereses, estrategias y escalas de acción que en ellos convergen, lo que otorga cierto interés al análisis de posibles contratendencias, resistencias y microdiferencias para dibujar con mayor precisión las nuevas formas emergentes y su lógica (Janoschka, 2011), revisando al mismo tiempo la supuesta banalización del espacio geográfico que, como en otros momentos del pasado, subyace a algunas de las propuestas presentadas.

\section{Los años de crecimiento y su impacto sobre el territorio metropolitano}

La región metropolitana madrileña ha conocido un constante crecimiento demográfico en las dos últimas décadas, pues los 4,9 millones de habitantes censados en 1991 se elevan a 6,5 millones al finalizar $2010(+30,5 \%)$. Esa tasa, superior al promedio nacional, le permite concentrar ya el $13,7 \%$ de la población residente en España, al tiempo que se sitúa en el tercer lugar dentro de la Unión Europea. En una sociedad con bajas tasas de fecundidad, donde las migraciones interregionales resultan bastante escasas desde hace décadas, buena parte de ese incremento se debió a la llegada de un importante volumen de inmigración exterior, atraída por una oferta laboral en expansión durante quince años, que incorpora hoy un elemento de especial significación a la nueva geografía humana de la aglomeración. Aunque las cifras oficiales infravaloran su presencia real, los 60.163 extranjeros contabilizados en 1991 pasaron a 282.870 en el año 2000, pero superan ya el millón (1.118.991) en 2010 , un $17,3 \%$ de la población total $(3,2 \%$ en el año 2000), con especial presencia de rumanos (222.528) y ecuatorianos (116.629), pero también de marroquíes (91.022), colombianos (68.915), peruanos (59.844), bolivianos (48.411) y chinos (45.710), que comparten hoy un territorio multicultural (Observatorio de la Inmigración, 2010).

Pero si la capacidad de Madrid para atraer población se ha mantenido en el tiempo, modificando la procedencia y características de los aquí instalados, mucho mayor resultó el aumento de las viviendas construidas en estos años. Su conversión en una forma de inversión aparentemente segura y rentable, ante la intensa especulación inmobiliaria favorecida por un crédito abundante y barato, permitió que las 30.000 viviendas anuales construidas hacia 1990 se convirtiesen en más de 50.000 en 2004, superando las 60.000 en 2005-06, momento culminante del proceso. En apenas dos décadas, las viviendas familiares en la región metropolitana pasaron de 1,9 a 2,9 millones, lo que supone una tasa anual acumulativa que casi duplicó la correspondiente a la población, con una estimación de más de 100.000 viviendas desocupadas en 2008 (Leal y Domínguez, 2009).

La conversión de Madrid en un magnífico ejemplo del proceso de financiarización y construcción de una sociedad de propietarios (López y Rodríguez, 2010) resulta indisociable del intenso dinamismo registrado por su economía desde mediados de los años noventa y hasta 2007, al combinarse las estrategias competitivas de actores privados y públicos en un contexto de globalización que favorecía los procesos de concentración metropolitana. Tanto en términos cuantitativos como cualitativos, la evolución registrada en esa larga fase expansiva situó a Madrid entre las regiones metropolitanas de éxito dentro de la Unión Europea, tal como oficializaron algunos informes de instituciones internacionales (OCDE, 2007), de lo que pueden ser buena muestra algunos indicadores seleccionados. 
Así, por ejemplo, el PIB madrileño aumentó en un $61,5 \%$ en valores constantes entre 1995 y 2010 , mientras el empleo metropolitano creció desde 2,1 a casi 3,5 millones entre 1995-2008 (+69,7\%), para retroceder desde esa fecha. El contrapunto fue una productividad prácticamente estancada, ante la elevada presencia de la construcción y ciertos servicios intensivos en mano de obra, pero con escaso valor añadido, frente al discurso oficial sobre la necesidad de una creciente eficiencia de la economía metropolitana. En consecuencia, la elevada tasa de paro existente en 1995 (20,5\%), tras la fase recesiva que vivió la economía española a principios de esa década, se redujo de forma rápida hasta el 6,3\% de promedio anual en 2007 , pese al aumento registrado por la población activa en esos mismos años debido a la progresiva incorporación de la mujer y, sobre todo, al elevado contingente migratorio. La paralela elevación de la renta media por habitante hasta los 18.175 euros en 2008, un $17,8 \%$ por encima del promedio nacional, supuso también un aumento en el consumo de todo tipo de bienes, apoyado además por un fuerte endeudamiento empresarial y familiar.

Más importante aún fue la consolidación de Madrid como principal centro concentrador de las funciones de comando y control en la Europa del Sur, tal como los numerosos estudios de la red Globalization and World Cities (GaWC) han demostrado. En una reciente publicación (Taylor et al., 2011), el análisis sobre localización de las sedes correspondientes a las 2.000 mayores empresas transnacionales sitúa a Madrid en el décimo lugar del mundo y tercero de Europa. Esa posición es muy similar a la que resulta de calcular su índice de conectividad, según el número y rango de los establecimientos pertenecientes a las 175 mayores empresas de servicios avanzados (puesto $11^{\circ}$ ) o las 75 principales entidades financieras (puesto $10^{\circ}$ ), lo que representó un importante salto cualitativo en poco más de una década (Sánchez et al., 2008) $)^{4}$.

\footnotetext{
4 En el ranking de conectividad internacional que encabeza Londres, la ciudad de Buenos Aires ocupa, por ejemplo, el puesto $16^{\circ}$, São Paulo el $21^{\circ}$, Ciudad de México el $24^{\circ}$ y Santiago de Chile el $41^{\circ}$ (Taylor et al., 2011: 24-25).
}

Su consolidación como centro de negocios, a la que se sumó un crecimiento también constante del turismo internacional, permitió que los 32,9 millones de pasajeros recibidos por el aeropuerto de Barajas en 2000 aumentasen a 48,3 millones en 2009, pasando del puesto $20^{\circ}$ al $11^{\circ}$ entre los principales aeropuertos del mundo según los informes anuales del Airports Council International. Finalmente, el informe European Cities Monitor, que identifica las ciudades del continente más valoradas para localizar un negocio a partir de diversos criterios cuantitativos y cualitativos, situó a Madrid en el octavo lugar en 2010 , frente al $17^{\circ}$ ocupado en 1990, para satisfacción de todos los aficionados a los rankings comparativos. En resumen, los optimistas diagnósticos oficiales de hace unos pocos años insistían en situar a Madrid entre las metrópolis ganadoras en el proceso de modernización capitalista, lo que se vio acompañado por una mutación no menos profunda y acelerada en la estructuración interna y los límites externos del territorio metropolitano, acorde con ese modelo de acumulación.

En primer lugar, se intensificó el ritmo de expansión de la mancha urbana, con la paralela transformación de un poblamiento históricamente concentrado y característico de la Europa mediterránea por otro más difuso. Apoyado por una fuerte inversión pública en todo tipo de infraestructuras que abrieron camino y facilitaron el negocio de los promotores privados, el urban sprawl se afirmó primero en la periferia suburbana del noroeste, correspondiente a las áreas de mayor calidad ambiental y nivel de renta. Pero en las dos últimas décadas las viviendas unifamiliares o colectivas de baja densidad en urbanizaciones dispersas se difundieron al conjunto metropolitano, provocando un rápido aumento del suelo ocupado por habitante (de 120 metros cuadrados en 1991 a 145 en 2005).

En la explicación de esta tendencia no puede negarse la influencia de factores de demanda, asociados al desarrollo de las clases medias y de unidades familiares más reducidas que apostaron por entornos menos congestionados que los característicos de las áreas urbanas centrales, a precios también inferiores. Pero el proceso es indisociable de la paralela desregulación del planeamiento que, 
apoyada en la Ley del Suelo de 1998, permitió convertir en urbanizables todos aquellos espacios no protegidos de forma explícita y convirtió a los promotores privados en principales agentes urbanizadores, mientras el sector público cargó con buena parte de los costes en infraestructuras y equipamientos derivados (Burriel, 2011). De este modo, tanto en la periferia de la ciudad de Madrid como de las restantes ciudades de la región metropolitana surgieron numerosas urbanizaciones, algunas de ellas cerradas, entremezcladas con parques industriales, comerciales, logísticos, científico-tecnológicos o de ocio, dando origen a un paisaje en apariencia caótico, aunque organizado a partir de múltiples lógicas particulares y estrategias de gobiernos locales no coordinados, ante la muy escasa voluntad reguladora del gobierno regional.

Esta difusión de la población y las actividades económicas a distancias cada vez mayores de la ciudad de Madrid puede sintetizarse al agregar la información por sucesivas coronas metropolitanas de diez kilómetros de anchura, aunque integrando las dos primeras dentro de la Ilamada Corona Metropolitana Funcional, que es donde se concentran las mayores tasas de crecimiento en las últimas décadas (Cuadro $N^{\circ} 1$ ).

\section{Cuadro $\mathrm{N}^{\circ} 1$}

Distribución de la población, los establecimientos con actividad económica y el empleo por coronas metropolitanas, 2009

\begin{tabular}{|l|r|r|r|}
\hline \multicolumn{1}{|c|}{ Unidades Territoriales } & $\begin{array}{c}\text { Porcentaje de } \\
\text { Población }\end{array}$ & $\begin{array}{c}\text { Porcentaje de } \\
\text { Establecimientos }\end{array}$ & $\begin{array}{c}\text { Porcentaje de } \\
\text { Empleos }\end{array}$ \\
\hline Madrid-capital & 50,1 & 57,9 & 62,8 \\
Corona Metropolitana Funcional $(<20 \mathrm{~km})$ & 35,6 & 29,3 & 28,7 \\
Tercera Corona $(20-29 \mathrm{~km})$ & 5,6 & 7,0 & 5,4 \\
Cuarta Corona $(30-39 \mathrm{~km})$ & 4,9 & 3,1 & 1,6 \\
Resto región metropolitana & 3,8 & 2,7 & 1,5 \\
Total región metropolitana & 100 & 100 & 100 \\
\hline
\end{tabular}

Fuente: Instituto Nacional de Estadísticas, 2009.

La ciudad de Madrid aún concentra la mitad de la población metropolitana, el 58\% de los establecimientos y cerca del $63 \%$ del empleo, lo que refleja las inercias asociadas a una aglomeración fuertemente monocéntrica hasta hace apenas medio siglo y las externalidades que aún se derivan de la proximidad física, más allá de la existencia de otras formas de proximidad (E.g. organizativa, cultural) que son posibles a mayor distancia. Las actividades más vinculadas a la centralidad como pueden ser las financieras o de servicios avanzados son las que más se resisten al traslado y mantienen a casi tres cuartas partes de sus trabajadores en inmuebles ubicados en la capital, pese a los recientes procesos de difusión hacia parques empresariales próximos, que apenas representan una cuarta parte del empleo total. El contraste con el empleo industrial, que solo mantiene un $37 \%$ del total en la ciudad central, evidencia lógicas diversas, analizadas en algún texto anterior (Méndez y Rodríguez, 2007).

Pese a tratarse de un proceso bastante más reciente que en otras aglomeraciones urbanas, la mejora de los transportes y comunicaciones, junto a una oferta de espacios de actividad cada vez más amplia, diversificada y de calidad, ha favorecido un traslado creciente 
de esas empresas industriales, logísticas, de distribución comercial y de ciertos servicios hacia aquellas ciudades en donde las estrategias de los promotores inmobiliarios se han combinado con políticas locales activas para atraer nuevas inversiones y empleos. Madrid tiende a convertirse, pues, en una región metropolitana policéntrica, aunque sin perder la jerarquización entre territorios, con una serie de subcentros localizados en las vías radiales de alta capacidad, que polarizan un volumen creciente de desplazamientos diarios. Es el caso de Alcobendas y San Sebastián de los Reyes en el sector norte, Alcalá de Henares, Coslada y Torrejón de Ardoz en el sector oriental, Getafe, Alcorcón, Leganés, Móstoles y Fuenlabrada en el sector sur, mientras Pozuelo de Alarcón y Las Rozas cumplen esa función en el sector oeste, concentrando en conjunto el 21,5\% del empleo total (Gallo et al., 2010).

Por último, aunque aquí se identifica la región metropolitana madrileña con el territorio integrado en su Comunidad Autónoma, es indudable que la región funcional desborda esos límites, sobre todo por el sur y el este (provincias de Toledo y Guadalajara), para hacerse presente en las provincias circundantes. Esa influencia supone tanto la implantación de empresas que pretenden reducir costes (E.g. suelo e inmuebles, salarios, impuestos), como la llegada de jóvenes residentes metropolitanos en busca de viviendas más baratas, lo que acentúa la integración, dinamismo y complejidad de esos espacios rururbanos en la aglomeración y la densidad de los movimientos pendulares diarios (Burns et al., 2009), pero manteniendo en todo caso su funcionalidad periférica.

\section{Contradicciones del crecimiento y crisis: vulnerabilidades del territorio metropolitano madrileño}

El tipo de crecimiento característico de este Madrid global generó diversas contradicciones y profundos contrastes, que alcanzan su culminación con la brusca ruptura producida en 2008. Puede así hablarse de una "crisis antes de la crisis", debido a "las contradictorias consecuencias de un determinado modelo social y urbano" (Observatorio Metropolitano, 2009: 13), del que pueden sintetizarse algunos de sus rasgos más representativos.

Por una parte, en los años de fuerte crecimiento económico buena parte del aumento registrado por el empleo se produjo en los segmentos más precarios, asociados al desarrollo del mercado de trabajo secundario, con lo que la tasa de temporalidad (porcentaje de contratos temporales sobre el total) se situaba en el $24,4 \%$ al finalizar 2007 , antes de que se iniciase el cambio de tendencia. Esa situación resultaba más acusada entre las mujeres, los jóvenes y los trabajadores con menor formación, pero se difundió también a los grupos más cualificados hasta convertirse en estructural, con una presencia máxima entre los inmigrantes. En un estudio realizado a un amplio colectivo de las cuatro nacionalidades más presentes en Madrid, pudo comprobarse que en su primer año de estancia tan solo un $6,3 \%$ declaraba haber tenido contrato fijo, por un $34,6 \%$ de contratos temporales y un $38,4 \%$ que trabajó sin contrato en la economía informal. En el momento de la investigación (2007), la situación había evolucionado hasta alcanzarse un $39,2 \%$ de contratos fijos, pero la suma de las otras dos situaciones $(41,9 \%)$ era aún superior, cualquiera que fuese su nivel formativo en origen (Méndez, 2008).

Rasgo esencial en la evolución de esos años fue el espectacular desarrollo del sector de la construcción, convertido en motor económico a costa de provocar importantes desajustes y un aumento de su vulnerabilidad. Apenas representaba el $7,2 \%$ del PIB en 1995, pero alcanzó el $10,6 \%$ en 2007 , dando empleo a casi el $12 \%$ de los ocupados en la región metropolitana. Al mismo tiempo, en los años de mayor auge inmobiliario (19982006) se construyeron 404.563 viviendas y su precio aumentó un promedio anual del 16,8\% en valores constantes, excluyendo del acceso a amplios sectores de la población y provocando un fuerte endeudamiento hipotecario ante la debilidad del mercado de alquiler. Ese proceso especulativo se alimentó, sobre todo, por la conversión de la vivienda en inversión segura que atrajo numerosos capitales, también internacionales, junto a un crédito barato y fácil ante la connivencia del sector financiero con los grupos inmobiliarios. Contó también con un apoyo directo de numerosos gobiernos 
locales, que aumentaban sus ingresos con las licencias de obras, junto a otro indirecto de los gobiernos estatal y regional (liberalización del suelo, desgravaciones fiscales...), más interesados en sus efectos multiplicadores sobre la economía a corto plazo que en valorar sus riesgos. Reflejo complementario de ese modelo de crecimiento disperso e intensivo en el consumo de suelo y energía, acentuado por el incremento de la movilidad forzada (García, 2008), fue un aumento superior al 5\% anual de la superficie artificializada, mientras el suelo calificado como urbano por el planeamiento lo hacía un 38\% entre 1992-2007. El indicador sintético de huella ecológica, calculado para el periodo 1995-2005 pasó de 5,58 a 6,75 $(+21,0 \%)$, mientras el cociente entre huella y biocapacidad lo hizo de 15,1 a 19,9 (+31,8\%), cuestionando así la sostenibilidad del modelo a largo plazo (De Santiago, 2008: 163).

La metamorfosis de la región metropolitana en estas dos décadas se acompañó de una creciente especialización y segmentación territorial, tanto socioeconómica como funcional, construyendo un mosaico heterogéneo en el que cada pieza se articula con el resto a partir de densas redes de flujos diarios. Esa tendencia se acentuó ante el predominio de la lógica inmobiliaria y los intereses de determinados actores privados, mientras unas administraciones públicas impregnadas de empresarialismo (Harvey, 2007) abandonaron su función reguladora en beneficio de la promoción y apoyo de tales procesos, sustituyendo la planificación por una multiplicación de proyectos parciales carentes de una perspectiva de conjunto.

Si se considera la distribución espacial de los diferentes grupos sociales, el elevado precio de la vivienda -asociado a otros aspectos como la calidad del entorno o su accesibilidad- acentuó su importancia como principal factor de selección, dentro del principio general de que unos residen donde quieren y otros donde pueden. En un marco de competencia desigual, las decisiones de instalación por parte de esos diferentes grupos resultaron acumulativas en el tiempo, tal como plantea la noción de path dependence (Martin y Simmie, 2008). Esto se reflejó en una reforzada concentración de la población con mayor nivel de renta en algunas ciudades metropolitanas situadas al norte (renta media disponible por habitante de 19.203 euros en 2008) y oeste
(23.125 euros) de la capital. Ese nivel de ingresos disminuye tanto al aumentar la distancia como, sobre todo, en dirección a las ciudades del sur y este de la aglomeración, que continúan alojando a las rentas inferiores, con niveles mínimos en sus municipios periféricos (renta media por habitante en torno a 13.000 euros).

Dentro de la capital (renta media de 19.390 euros), el contraste entre los barrios del noroeste y sureste no solo se mantuvo, sino que se vio reforzado, si bien el cambio de escala introducía mayor complejidad debido al asentamiento de un elevado volumen de inmigrantes y grupos con escasos ingresos en algunas áreas deterioradas del centro o próximas a él, en viviendas de baja calidad construidas hace al menos un siglo para alojar a los inmigrantes que entonces llegaban de otras regiones españolas (E.g. Lavapiés, Usera, Tetuán, Puente de Vallecas) y cuyos descendientes fueron progresivamente sustituidos. Por el contrario, otros enclaves próximos al eje de negocios del paseo de la Castellana o en pleno centro histórico conocieron procesos de gentrificación y asentamiento de profesionales cualificados, atraídos por el ambiente cultural y las amenidades urbanas.

También se consolidaron estrategias de localización más selectivas para las diversas actividades económicas, tanto en sentido centro-periferia como noroeste-sureste. Las nuevas actividades industriales y, sobre todo, logísticas y de gran distribución se localizaron, principalmente, en un arco que por el este y sur envuelve la capital y las ciudades próximas, con las mayores tasas de crecimiento de la última década localizadas a más de 30 kilómetros de la capital. No obstante, la creciente segmentación espacial de la cadena de valor favoreció que determinadas sedes o centros de I+D de grandes empresas industriales se ubicaran en parques empresariales del norte metropolitano e, incluso, en ciertos distritos de la capital, donde su presencia apenas se asocia ya con actividades fabriles de producción.

Como contrapunto, la resistencia de los servicios avanzados $-y$, en buena medida, de las sedes financieras y de seguros- a abandonar la ciudad de Madrid reforzó incluso su presencia en sus áreas centrales más valoradas, entre el paseo de la Castellana y el aeropuerto 
de Barajas, avanzando lentamente hacia las ciudades próximas del sector nordeste. Buena prueba de ello es que la máxima valoración actual corresponde al Cuatro Torres Business Area (CTBA), nuevo espacio corporativo y simbólico al norte de la capital, sobre terrenos de la antigua Ciudad Deportiva del Real Madrid, pero aún dentro de su término municipal.

El shock financiero internacional a mediados de 2008 provocó una brusca detención del proceso, al desaparecer el crédito barato que lo alimentaba. El deterioro de la situación resultó particularmente grave en áreas urbanas que, como Madrid, habían basado buena parte de su crecimiento en actividades de baja productividad y empleos poco cualificados, descuidando una industria en progresivo declive y con unos servicios avanzados incapaces, por sí solos, de frenar el proceso. Acabó así un periodo expansivo que algunos creyeron inagotable y se inició otra fase del ciclo económico para la que algunos indicadores laborales son el mejor exponente de la situación.

Así, por ejemplo, entre el último trimestre de 2007 y el de 2010 la ocupación en la región metropolitana se redujo en 175.400 trabajadores, mientras los desempleados aumentaron en 332.600 debido al aumento de la población activa, con una tasa de paro que del 6,4\% se elevó al 15,8\% según la Encuesta de Población Activa (Figura No 1). Tal como se repite en cada fase recesiva, ese aumento del desempleo afectó, sobre todo, a los jóvenes menores de 25 años, cuya tasa de paro casi duplica ese promedio, así como a los inmigrantes $(23 \%)$, mientras fue menor en el caso de las mujeres, al concentrarse las pérdidas en la construcción y la industria (Fundación Sindical de Estudios, 2010). Aún no existen análisis publicados sobre el impacto territorial de esta crisis que, más allá de una simple onda en la evolución cíclica del sistema, parece suponer cambios estructurales en la posición relativa de los países europeos dentro de la división internacional del trabajo y cuestiona en particular modelos de crecimiento como el aquí esbozado. Pero los datos, por el momento escasos, de que se dispone parecen confirmar unos efectos más acusados en aquellas áreas de la aglomeración con mayor presencia de grupos socioprofesionales vinculados a los empleos más vulnerables y a las actividades con menor capacidad competitiva.

Figura $N^{\circ} 1$

Evolución del desempleo y la tasa de paro en la región metropolitana de Madrid, 2006-2010 (datos trimestrales)

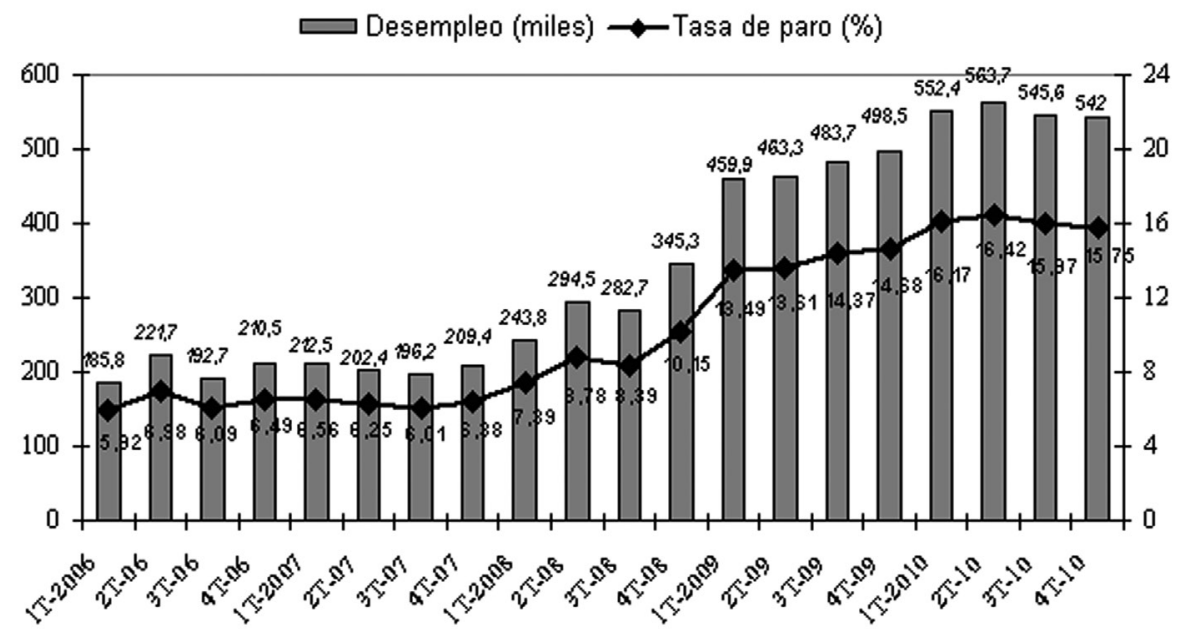

Fuente: INE, 2010. 
En resumen, con el esquematismo propio de una panorámica de conjunto, las páginas anteriores han pretendido describir y comprender algunas claves de la trayectoria seguida por Madrid en el marco de la globalización capitalista. Pero solo cuando se aproxima el foco de atención a espacios, sectores o grupos sociales concretos se obtiene una visión en profundidad del sentido de tales transformaciones. Por ese motivo, el estudio de las características, evolución reciente, distribución espacial y calidad del empleo en la Ilamada economía del conocimiento resulta hoy un buen exponente de los logros, limitaciones y costes de ese proceso, así como de la nueva estructura espacial emergente en el interior de la región metropolitana y las nuevas formas de desigualdad resultantes, que exigen nuevos indicadores para identificarlas y actuar sobre ellas.

\section{Economía del conocimiento: importancia actual y estrategias de localización}

Las regiones metropolitanas europeas se enfrentan hoy a la creciente competencia derivada del actual contexto de globalización y del proceso de integración regional, a los que, en casos como el de Madrid, se suma el agotamiento de su anterior modelo de crecimiento. De ahí la difusión de un discurso que insiste en la necesidad de desarrollar una economía más intensiva en la generación y uso del conocimiento, asociada a un mayor esfuerzo innovador, a empleos de alta cualificación y más estables, así como con un menor impacto ambiental, que se enfrente también de modo más eficaz al riesgo de deslocalización, dando origen a lo que algunos autores definen como ciudades del conocimiento (Ergazakis et al., 2004; Winden et al., 2007).

La progresiva inclusión de esa economía del conocimiento en las agendas de investigación actuales, pese a que el origen del concepto se remonte varias décadas, intenta identificar la importancia, características, dinamismo reciente, pautas de localización e impactos territoriales de aquellas actividades con mayor contenido en conocimiento, pero también de aquellas ocupaciones más cualificadas y con exigencias formativas superiores, cualquiera que sea el sector en que se integren, así como las formas de gobernanza y las políticas más eficaces para su promoción. Su presencia se utiliza también como indicador de las nuevas formas de jerarquización y desigualdad en el interior de los sistemas urbanos (Scott, 2009), aportando una panorámica transversal de las economías metropolitanas complementaria a las clásicas divisiones sectoriales.

Una primera aproximación al mapa de la economía del conocimiento en Madrid puede hacerse, pues, al identificar la presencia de aquellas actividades más intensivas en el uso de un recurso siempre presente, pero ahora valorado como estratégico, que se pretende producir, transferir y aplicar de modo sistemático. En las clasificaciones hechas por diversos organismos internacionales aquí suelen incluirse cuatro tipos de actividades. Están, en primer lugar, las industrias de alta intensidad tecnológica (farmacéutica y biotecnología, electrónica e informática, aeronáutica, instrumental científico...) y toda una serie de servicios avanzados a empresas, junto a finanzas y seguros, educación y sanidad. La mayor novedad de los últimos años radica en la inclusión de aquellas actividades que proveen de bienes y servicios culturales, protegidos a menudo por leyes de propiedad intelectual, entre los que se cuentan algunos de larga tradición (edición de libros, producción musical y audiovisual, archivos, bibliotecas y museos, actividades artísticas), junto a otros relacionados con las nuevas tecnologías digitales (productos multimedia e infográficos, videojuegos, etc.). El concepto de ciudad creativa presta especial atención a la presencia de estas últimas, junto a servicios como los de arquitectura o publicidad (Scott, 2006; Cooke \& Lazzeretti, 2008).

La región metropolitana de Madrid es, con diferencia, la principal aglomeración de actividades intensivas en conocimiento en España. El conjunto de externalidades asociadas a la aglomeración, la acumulación de inversiones públicas derivadas de la capitalidad (universidades, centros de investigación, museos y otros equipamientos culturales...), la presencia de capital humano altamente cualificado o la existencia de clusters empresariales consolidados en el tiempo son algunas de las claves generadoras de ventajas 
competitivas que justificaron su crecimiento en las últimas décadas.

Según datos de la Tesorería General de la Seguridad Social para 2009, las actividades intensivas en conocimiento suponen un total de 41.043 empresas y 859.655 empleos, lo que equivale al $23,5 \%$ del total existente en España, muy por encima de Barcelona $(15,9 \%)$, que es la segunda aglomeración del país (Trullén et al., 2002). Dentro del empleo total de la región metropolitana, esa cifra equivale ya al $30,5 \%$, alcanzando el $33,5 \%$ en el caso de la ciudad de Madrid, mientras en el resto de la aglomeración disminuye al 25,4\% (Cuadro $N^{\circ} 2$ ). No se trata, por tanto, de unas actividades marginales, sino que su presencia es ya bastante destacada.

Cuadro $\mathrm{N}^{\circ} 2$

Actividades intensivas en conocimiento en la región metropolitana de Madrid

\begin{tabular}{|l|r|r|r|r|}
\hline Actividades & Empresas & $\begin{array}{r}\text { Porcentaje } \\
\text { total }\end{array}$ & Empleos & $\begin{array}{r}\text { Porcentaje } \\
\text { total }\end{array}$ \\
\hline Industrias high tech & 511 & 0,2 & 36.631 & 1,3 \\
Finanzas y seguros & 2.357 & 1,0 & 112.358 & 4,0 \\
Servicios avanzados a las empresas & 22.146 & 9,1 & 348.295 & 12,4 \\
Educación & 5.585 & 2,3 & 140.070 & 5,0 \\
Sanidad & 5.213 & 2,1 & 133.989 & 4,7 \\
Industrias y servicios culturales & 5.231 & 2,12 & 88.312 & 3,1 \\
Total Economía del Conocimiento & 41.043 & 16,9 & 859.655 & 30,5 \\
Restantes actividades económicas & 201.900 & 83,1 & 1.957 .989 & 69,5 \\
TOTAL & 242.943 & 100 & 2.817 .644 & 100 \\
\hline
\end{tabular}

Fuente: Tesorería General de la Seguridad Social, 2009.

Los servicios avanzados representan casi la mitad de esa ocupación (348.295), seguidos de lejos por la educación, la sanidad $y$ el sector financiero y de seguros, que en conjunto suman poco más que la cifra anterior (386.417). En posición más modesta se sitúa el sector cultural con 88.312 empleos, mientras las industrias high tech cuentan con menos de la mitad de esa cifra (36.631). En resumen, los servicios representan lo esencial de la economía del conocimiento en Madrid, pero mientras los de carácter público vinculados a la educación y la sanidad suponen algo menos de una tercera parte, los de carácter privado relacionados con el mundo de las finanzas o los servicios de valor añadido a empresas son ya ampliamente predominan- tes, como corresponde a su posicionamiento internacional.

Se trata, pues, de un conjunto heterogéneo, formado por actividades que han mostrado un comportamiento reciente también contrastado pero menos favorable de lo que cabría esperar si se atiende a las supuestas ventajas competitivas derivadas de la propia escala urbana. De este modo, entre 2000 y 2006 el empleo en estos sectores aumentó un $28,8 \%$, en consonancia con una fase de expansión económica. Esa tasa fue ligeramente inferior a la registrada por la economía metropolitana en su conjunto $(29,7 \%)$, con los servicios avanzados a las empresas (36,8\%), la sanidad $(35,1 \%)$ y la educación $(32,2 \%)$ 
como los más dinámicos. No obstante, con la profunda crisis posterior estas actividades no solo se mostraron incapaces de mantener ese crecimiento, sino que entre 2006 y 2009 perdieron empleos a un ritmo muy superior $(-19,9 \%)$ al registrado por el sistema productivo regional en su conjunto $(-5,3 \%)$ debido, sobre todo, al fuerte retroceso de una parte de los servicios avanzados $(-39,5 \%)$ muy vinculados al proceso de financiarización y al freno sufrido por la demanda empresarial. También perdieron efectivos las aquí siempre débiles industrias de alta tecnología $(-13,2 \%)$, mientras el empleo público en educación y sanidad se estancaba. Puede cuestionarse, por tanto, que la economía del conocimiento tienda a crecer en las regiones metropolitanas al margen de los ciclos económicos en ausencia de políticas explícitas de impulso.

Pero la emergencia de estas actividades también contribuye de forma significativa a transformar los modelos de organización territorial heredados y adecuarlos a esta nueva fase de desarrollo capitalista. Las estrategias de localización que aplican las empresas e instituciones aquí integradas cobran creciente importancia para dinamizar determinadas áreas de la aglomeración, que muestran ahora una especial capacidad de atracción, mientras su ausencia en otras es una de las causas de su declive o de una especialización en tareas y empleos de menor rango.

El primer rasgo a destacar es la elevada concentración territorial que aún muestran si se analizan de forma conjunta. Frente a los procesos de difusión espacial de la industria, la logística o las grandes superficies comerciales, las actividades más relacionadas con la economía del conocimiento muestran una distribución mucho más concentrada, en donde la capacidad de atracción ejercida por la ciudad central apenas parece debilitarse pese al incremento de las deseconomías externas en un espacio de alta densidad y elevados costes. Un $70,2 \%$ de las empresas y el $69,2 \%$ de los empleos siguen localizados en la capital, con una especial polarización en torno al centro de negocios y los barrios que hacia el norte y el aeropuerto amplían el perímetro de esa área de forma progresiva, pero sin provocar apenas su sustitución por nuevos subcentros suburbanos de verdadera entidad (Cuadro No 3 ).

Cuadro $\mathrm{N}^{\circ} 3$

Concentración de la economía del conocimiento por coronas metropolitanas

\begin{tabular}{|l|r|r|}
\hline Unidades Territoriales & \multicolumn{1}{|c|}{$\begin{array}{c}\text { Porcentaje } \\
\text { Empleo total }\end{array}$} & $\begin{array}{c}\text { Porcentaje Empleo } \\
\text { en Economía del } \\
\text { Conocimiento }\end{array}$ \\
\hline Madrid-capital & 62,8 & 69,2 \\
Corona Metropolitana Funcional $(<20 \mathrm{~km})$ & 28,7 & 29,6 \\
Tercera Corona $(20-29 \mathrm{~km})$ & 5,4 & 0,5 \\
Cuarta Corona $(30-39 \mathrm{~km})$ & 1,6 & 0,6 \\
Resto región metropolitana & 1,5 & 0,1 \\
Total región metropolitana & 100 & 100 \\
\hline
\end{tabular}

Fuente: Tesorería General de la Seguridad Social, 2009.

En ese sentido, sin negar la tendencia general ya señalada hacia una estructura metropolitana más policéntrica, no debe ignorarse la influencia que aún ejercen los modelos urbanos heredados en la definición de las actuales trayectorias espaciales. En aglomeraciones metropolitanas de tradición fuertemente monocéntrica, como es el caso 
de Madrid, la existencia de suelo disponible para nuevos desarrollos en la capital y, sobre todo, el valor simbólico que aún se otorga a algunas de esas áreas centrales actúa como recurso intangible que frena el traslado de la mayor parte de estas empresas, en especial sus centros de decisión y representación, o que están en contacto directo con los clientes. Algo similar ocurre con las instituciones universitarias y científicas o los grandes iconos culturales, que permanecen en la ciudad de Madrid o, a lo sumo, en unas pocas ciudades metropolitanas contiguas, sin apenas deslocalizarse hacia los espacios suburbanos o periurbanos. Así, por ejemplo, el uso de coronas metropolitanas de diez kilómetros de radio permite comprobar que las ciudades situadas en las dos primeras suman un 29,6\% de los ocupados, frente a proporciones irrelevantes en el resto. También que su importancia relativa muestra una similar disminución con la distancia, aunque con gradiente es menos acusado: si en la capital estas actividades representan un tercio del empleo total $(33,5 \%)$, en la primera corona se reduce al $28,3 \%$, para mantenerse en torno al $15 \%$ en las tres siguientes y disminuir al $12 \%$ en la más alejada.

Pero la segmentación del territorio metropolitano que favorecen las actividades intensivas en conocimiento se aprecia mejor al considerar la especialización relativa de los municipios de la región. Aquellos que muestran proporciones superiores al promedio, reflejadas en cocientes de localización superiores a la unidad, se sitúan casi exclusivamente en los sectores metropolitanos de más alta valoración social y ambiental, al noroeste de la capital, que hasta hace tres décadas mantenían una función residencial ahora complementada con nuevas áreas empresariales, campus universitarios y financieros, instituciones educativas y de salud privadas, etc. (Figura $\mathrm{N}^{\circ} 2$ ). Este es el caso de Alcobendas, Tres Cantos y San Sebastián de los Reyes, en el norte, o Las Rozas, Pozuelo de Alarcón y Boadilla del Monte, en el oeste. Estas dos últimas ciudades son las que presentan las proporciones más altas de empleo en estas actividades con relación al total $(50,8 \%$ y $56,8 \%$ respectivamente), muy por encima de la propia capital. Esa situación contrasta con su escasa importancia en las antiguas áreas industriales y obreras del sur y este metropolitanos, poco atractivas para este tipo de actividades, donde tan solo algunos gobiernos locales fueron capaces en estos años de impulsar estrategias de revitalización que incluían la atracción de universidades públicas, la promoción de parques tecnológicos, centros culturales, etc., con Getafe como ejemplo destacado. 
Figura $\mathrm{N}^{\circ} 2$

Especialización municipal en economía del conocimiento: cocientes de localización

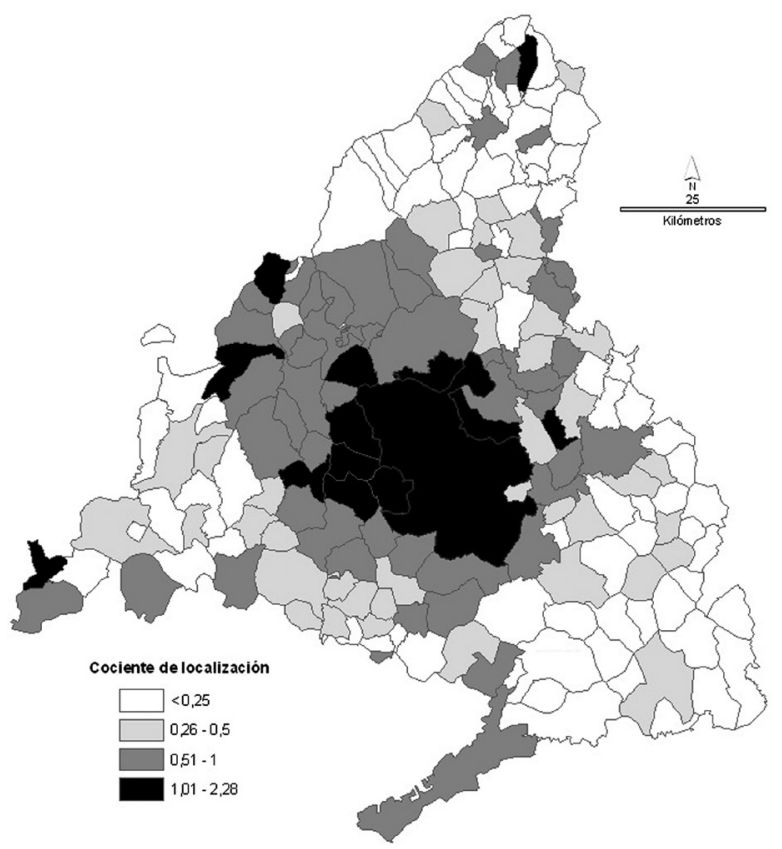

Fuente: Elaboración propia a partir de datos de la Seguridad Social, 2009.

Un último rasgo a destacar es la diversidad de pautas de localización según tipos de actividades, lo que demuestra la persistencia de dinámicas de concentración en determinadas áreas, no solo por acumular factores de atracción específicos, sino también por el desarrollo de interrelaciones entre las empresas y la consiguiente creación de clusters localizados, que intercambian conocimiento tácito a partir del establecimiento de dinámicas de proximidad física, organizativa y cultural (Bathelt et al., 2004). Los mapas de la figura 3 sintetizan esos comportamientos mediante cocientes de localización, según su proporción de empleo en el total municipal respecto al promedio de cada actividad en la aglomeración.

Así se constata, por ejemplo, que las industrias de alta intensidad tecnológica han abandonado ya la capital, pero se concentran en las ciudades localizadas en los ejes radia- les que parten de la misma. Por el contrario, tanto los servicios avanzados como la educación superior tienen una presencia relativa destacada en la franja suburbana occidental, que cuenta también con niveles de renta más elevados. Por su parte, tanto las actividades financieras y de seguros como las relacionadas con la salud son las que mantienen una mayor resistencia a abandonar la ciudad de Madrid, descentralizando tan solo sus centros de trabajo de menor rango hacia municipios contiguos (junto a la presencia de residencias sanitarias en el área de la sierra de Guadarrama). El sector cultural es, finalmente, el que muestra una distribución más compleja, debido a la variedad de actividades que engloba y a la importancia que en algunos casos han tenido las políticas desarrolladas por los gobiernos locales en la promoción de equipamientos culturales, frente a la permanencia de las grandes instituciones e industrias culturales en la capital. 


\section{Contratos a profesionales de alta cualificación: una perspectiva complementaria}

Una visión complementaria a esta es la que ofrece la distribución de los trabajadores con alta cualificación profesional y nivel formativo, cualquiera que sea el sector de actividad de la empresa. En ese aspecto, la referencia de Florida (2002) a la clase creativa es la más repetida en los últimos años, pese a lo discutible que resulta otorgar identidad de clase a ese heterogéneo grupo y asociar la creatividad con un determinado puesto de trabajo o un nivel educativo, aspectos no obstante ajenos a nuestros objetivos y tratados por otros autores (Peck, 2005). A diferencia de la perspectiva anterior, para la que el conocimiento es un atributo de las empresas, aquí lo es de las personas, que lo adquieren principalmente a través del sistema educativo. Por ese motivo, la presencia de profesionales universitarios, directivos o gerentes en la población ocupada se considera un indicador relevante, no necesariamente paralelo a la importancia de las actividades intensivas en conocimiento.

Si el estudio territorializado de la economía del conocimiento a partir de las actividades resulta aún poco frecuente, aún lo es menos el planteado desde este otro enfoque. Una de las razones en el caso español es la inexistencia de esta información a escala local salvo en el caso de los censos, el último de los cuales es de 2001 y resulta, por tanto, poco adecuado para el análisis propuesto. Por ese motivo se ha explotado por primera vez a escala intrametropolitana la información del Instituto Nacional de Empleo (INEM) sobre todos los contratos registrados durante el periodo 2005-2009, identificando el municipio metropolitano donde se localizan las empresas o instituciones que los firmaron, así como las ocupaciones para las que se contrató a los trabajadores y las principales características de los contratos.

Se trata de una información que exige tener en cuenta sus evidentes diferencias con otras fuentes más habituales, desde varios puntos de vista: (i) Las cifras que se presentan no son stocks sino flujos, pues identifican el número de contratos firmados y no el volumen final de empleo, ya que un mismo trabajador puede haber firmado varios en el periodo analizado; (ii) Ofrece una perspectiva dinámica sobre la importancia de cada tipo de ocupación profesional en los contratos firmados durante un lustro que integra años de expansión y de recesión, lo que permitiría observar el desigual impacto de la actual crisis en un estudio más detallado que el aquí realizado; (iii) Posibilita también relacionar la cualificación profesional con la estabilidad o precariedad de los contratos firmados, aspecto relevante para observar la nueva estructura socioespacial metropolitana emergente desde una perspectiva inexplorada hasta el momento.

Durante el periodo 2005-2009 se firmaron en la región metropolitana de Madrid un total de 11,2 millones de contratos, con un máximo registrado en el año 2006 (2,6 millones) y un fuerte descenso posterior hasta los 1,7 millones en 2009. Si utilizamos la clasificación de actividades, las intensivas en conocimiento concentraron algo más de una tercera parte del total $(35,5 \%)$. Esa importancia se reduce de forma significativa si se consideran los contratos a profesionales de alta cualificación, que supusieron el 18,0\% del total. Por su parte, si se consideran los contratos a titulados universitarios de primer o segundo ciclo, la cifra resultó algo inferior, con un $17,2 \%$ del total. Se trata, por tanto, de indicadores complementarios pero heterogéneos, que pueden modificar el diagnóstico final según se utilicen unos $u$ otros.

Centrando la atención en el tipo de ocupaciones para las que se contrató, el cuadro 4 plantea una desagregación a partir de la Clasificación Nacional de Ocupaciones (CNO) que detalla las más cualificadas, que son las que aquí interesa destacar, cruzando su volumen e importancia relativa con la duración indefinida o temporal del contrato y el carácter de la jornada laboral, a tiempo completo o parcial. Pueden destacarse algunos resultados que matizan los argumentos presentados hasta el momento (Figura $N^{\circ} 3$ y Cuadro $N^{\circ} 4$ ). 
Figura $\mathrm{N}^{\circ} 3$

Especialización según tipos de actividades intensivas en conocimiento

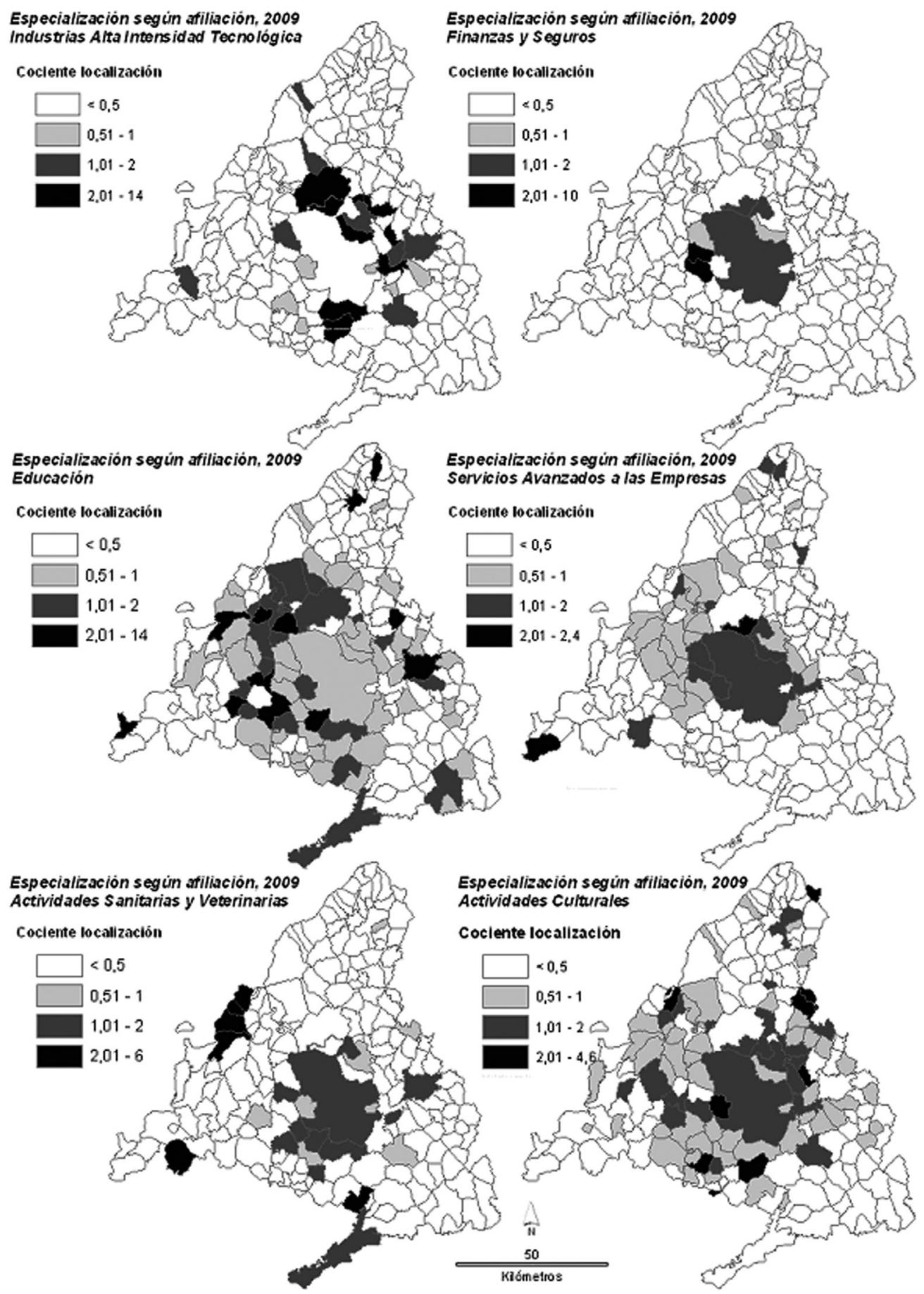

Fuente: Elaboración propia a partir de datos de la Seguridad Social, 2009. 


\section{Cuadro $\mathrm{N}^{\circ} 4$}

Nuevos contratos firmados en la región metropolitana de Madrid según ocupación y tipo de contratación, 2005-2009

\begin{tabular}{|c|c|c|c|c|c|c|c|}
\hline Tipo de ocupación & $\begin{array}{c}\mathrm{N}^{\circ} \text { de } \\
\text { contratos }\end{array}$ & $\begin{array}{c}\text { Porcentaje } \\
\text { Total }\end{array}$ & $\begin{array}{l}\text { Indefinidos } \\
\text { Tiempo } \\
\text { completo }\end{array}$ & $\begin{array}{c}\text { Indefinidos } \\
\text { tiempo } \\
\text { parcial }\end{array}$ & $\begin{array}{c}\text { Temporales } \\
\text { tiempo } \\
\text { completo }\end{array}$ & $\begin{array}{c}\text { Temporales } \\
\text { tiempo } \\
\text { parcial }\end{array}$ & Formativos \\
\hline Directivos y Gerentes & 68.818 & 0,6 & 2,7 & 0,7 & 0,3 & 0,1 & 0,5 \\
\hline Profesionales Ciencias físicas/Sanidad & 181.263 & 1,6 & 5,0 & 1,2 & 1,0 & 0,7 & 14,6 \\
\hline Profesionales de la Enseñanza & 190.626 & 1,7 & 0,7 & 2,4 & 0,7 & 4,3 & 2,4 \\
\hline Profesionales Derecho y CCSS/Humanas & 100.426 & 0,9 & 3,3 & 0,8 & 0,5 & 0,4 & 6,7 \\
\hline Escritores & 188.524 & 1,7 & 0,5 & 0,2 & 2,7 & 0,3 & 1,7 \\
\hline Profesión. Universitarios 1er ciclo & 263.384 & 2,3 & 4,4 & 1,6 & 2,1 & 1,7 & 8,4 \\
\hline Técnicos de Apoyo & 1.034 .392 & 9,2 & 14,4 & 6,5 & 8,7 & 7,9 & 14,7 \\
\hline Total profesionales cualificados & 2.027 .433 & 18,0 & 31,0 & 13,4 & 16,0 & 15,4 & 49,0 \\
\hline Trabajadores de servicios & 4.307 .380 & 38,3 & 39,1 & 57,1 & 31,1 & 50,6 & 28,4 \\
\hline Trabajadores de producción & 1.454 .106 & 12,9 & 10,7 & 2,8 & 18,9 & 2,3 & 21,7 \\
\hline Conductores & 339.908 & 3,0 & 4,7 & 4,6 & 3,3 & 1,5 & 0,2 \\
\hline Trabajadores no cualificados & 3.124 .385 & 27,8 & 14,5 & 22,1 & 30,7 & 30,2 & 0,5 \\
\hline Total contratos & 11.253 .212 & 100 & 100 & 100 & 100 & 100 & 100 \\
\hline
\end{tabular}

Fuente: Instituto Nacional de Empleo, 2009.

La mitad de los poco más de dos millones de contratos firmados para las ocupaciones profesionales más cualificadas correspondieron a técnicos de apoyo, que es el grupo que presenta una cualificación inferior, muy por delante de los profesionales con titulación universitaria de primer ciclo, los profesionales de la enseñanza, los escritores o los profesionales vinculados a las ciencias físico-naturales, sanitarias, sociales o humanas, quedando en último término los directivos y gerentes. Si se utiliza un criterio más exigente, que excluye al personal técnico auxiliar, las ocupaciones de alta cualificación representaron menos de una décima parte de todos los contratos, lo que permite recordar que buena parte de los empleos en actividades de alta intensidad en conocimiento se ocupan de tareas con limitadas exigencias formativas. Por el contrario, fue muy superior el volumen de contratos para ocupaciones de media o baja cualificación relacionadas con los servicios (4,3 millones, un $38,3 \%$ del total), que triplicaron los correspondientes a trabajadores de producción $(1,5 \mathrm{mi}$ llones), pese al dinamismo de la construcción hasta 2008. A este último sector correspondieron también muchos de los contratos para trabajadores no cualificados (peones), que aun superaron ampliamente la cuarta parte del total $(27,8 \%)$.
Una posible explicación de la escasa importancia relativa del capital humano más cualificado podría relacionarse con la mayor estabilidad de sus empleos, que reduce su presencia en las nuevas contrataciones. Esa posibilidad parece confirmarse al comprobar que su proporción sobre los 1,5 millones de contratos de duración indefinida y a tiempo completo firmados en esos cinco años ascendió al $31,0 \%$, muy por encima de su peso relativo en el conjunto $(18,0 \%)$ y duplicando el alcanzado entre los contratos temporales a tiempo completo $(16,0 \%)$ o parcial $(15,4 \%)$. Pero esa mejor situación en términos comparativos no puede ignorar que, pese a la alta cualificación exigida, tan solo una cuarta parte $(25,6 \%)$ correspondió a contratos indefinidos, frente a tres cuartas partes $(74,1 \%)$ de contratos temporales y un $0,3 \%$ de contratos formativos (becarios, médicos residentes...). Ese valor medio resulta de agregar situaciones muy diferentes, pues la contratación indefinida resultó elevada entre directivos y gerentes $(62,6 \%)$ mientras, en el extremo opuesto, resultó muy escasa entre profesionales de la enseñanza $(11,6 \%)$ y escritores $(4,0 \%)$, poniendo de manifiesto la limitada importancia del nivel de conocimiento de los contratados frente al tipo de relaciones laborales y grado de sindicalización característico de cada sector profesional. 
Por último, el análisis de la distribución espacial de las empresas e instituciones donde trabajaron los nuevos contratados confirma la nueva división espacial del trabajo que conlleva la expansión de esta economía del conocimiento. Para reflejar esa lógica espacial, el Cuadro $N^{0} 4$ muestra la especialización según tipos de ocupación correspondiente a la ciudad de Madrid y las otras trece ciudades donde a lo largo de esos cinco años se firmaron más de 100.000 contratos, identificando el sector metropolitano en que se localizan. Para ofrecer una imagen gráfica de más fácil lectura se sustituyeron los valores numéricos de los cocientes de localización por un sombreado que identifica aquellas ocupaciones y ciudades donde esos cocientes superan la unidad.

La ciudad capital muestra una proporción de nuevos contratos superior al promedio en todas las ocupaciones de alta cualificación y en aquellas vinculadas al trabajo en los servicios, reflejo tanto de su intensa terciari- zación como de una primacía funcional aún incontestada. Las ciudades contiguas que se localizan al oeste (Pozuelo de Alarcón) y norte (Alcobendas) se caracterizan por una especialización en el mismo tipo de ocupaciones, que también predominan en otras ciudades noroccidentales más alejadas (San Sebastián de los Reyes, Tres Cantos, Las Rozas), con un perfil más diversificado por la destacada presencia de actividades productivas, grandes centros comerciales y parques de ocio. Como contrapunto, en las ciudades del sur y este metropolitanos las contrataciones se concentraron en ocupaciones de cualificación media o baja, reflejo del tipo de empresas (industriales, logísticas, de construcción...) aún predominantes. Tan solo en casos como los de Alcalá de Henares, Getafe, Alcorcón o Móstoles la presencia de universidades públicas, grandes hospitales o algún parque tecnológico justifica una proporción destacada de contratos a profesionales con titulación superior.

\section{Cuadro $\mathrm{N}^{\circ} 4$}

Especialización de los nuevos contratos según tipos de ocupaciones en las principales ciudades de la región metropolitana de Madrid

\begin{tabular}{|c|c|c|c|c|c|c|c|c|c|c|c|c|c|c|}
\hline \multirow[b]{2}{*}{ Tipos de Ocupaciones } & \multirow[b]{2}{*}{ 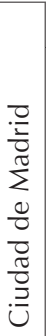 } & \multicolumn{3}{|c|}{ NORTE } & \multicolumn{2}{|c|}{ OESTE } & \multicolumn{3}{|c|}{ ESTE } & \multicolumn{5}{|c|}{ SUR } \\
\hline & & $\begin{array}{l}0 \\
\frac{\pi}{0} \\
\frac{C}{0} \\
\frac{0}{0} \\
\frac{U}{<}\end{array}$ & 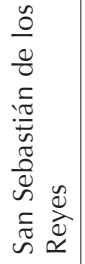 & 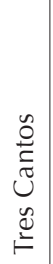 & $\begin{array}{l}0 \\
0 \\
0 \\
\frac{0}{2} \\
0 \\
0 \\
0 \\
0 \\
0 \\
\frac{0}{2} \\
0 \\
0\end{array}$ & $\begin{array}{l}\tilde{J} \\
N \\
\tilde{N} \\
\tilde{N} \\
\tilde{J}\end{array}$ & $\frac{\frac{\pi}{0}}{\frac{\pi}{n}} \frac{0}{0}$ & 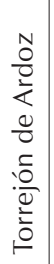 & 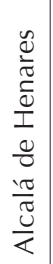 & $\begin{array}{l}0 \\
0 \\
\frac{0}{0} \\
\frac{U}{<}\end{array}$ & 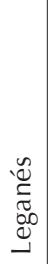 & 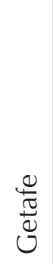 & 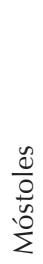 & $\begin{array}{l}\frac{\pi}{0} \\
\frac{\pi}{0} \\
\frac{\pi}{0} \\
\frac{0}{c} \\
\frac{0}{U}\end{array}$ \\
\hline \multicolumn{15}{|l|}{ Directivos y gerentes } \\
\hline \multicolumn{15}{|c|}{ Profesionales de Ciencias físicas/Sanidad } \\
\hline \multicolumn{15}{|l|}{ Profesionales de la Enseñanza } \\
\hline \multicolumn{15}{|c|}{ Profesionales Derecho y CCSS/Humanas } \\
\hline \multicolumn{15}{|l|}{ Escritores } \\
\hline \multicolumn{15}{|c|}{ Profesionales universitarios de 1 er ciclo } \\
\hline \multicolumn{15}{|l|}{ Técnicos de apoyo } \\
\hline \multicolumn{15}{|l|}{ Trabajadores de los servicios } \\
\hline \multicolumn{15}{|l|}{ Trabajadores del sector agrario } \\
\hline \multicolumn{15}{|l|}{ Trabajadores de la construcción } \\
\hline \multicolumn{15}{|l|}{ Trabajadores de la industria } \\
\hline \multicolumn{15}{|l|}{ Conductores } \\
\hline Trabajadores no cualificados & & & & & & & & & & & & & & \\
\hline
\end{tabular}

Fuente: Instituto Nacional de Empleo, 2009. 


\section{Consideraciones finales}

Tal como afirma Capel (2009: 10), "está ampliamente reconocido que las grandes ciudades son las áreas de la innovación, del desarrollo industrial y de la concentración de servicios especializados. En ellas se reúne la mayor cantidad de empleos, especialmente los más intensivos en conocimiento". El proceso de globalización, al que comenzó a ponerse nombre hace ahora dos décadas, ha acentuado esa tendencia a la centralización del poder en un número reducido de grandes aglomeraciones urbanas que, al mismo tiempo, se extienden sobre el territorio hasta hacer cada vez más difícil intentar dibujar sus límites y lo ocurrido en Madrid es coherente con esas tendencias. Desde mediados de los años noventa y hasta 2008 aquí se registró un intenso proceso de crecimiento impulsado por factores de carácter estructural favorables a la aglomeración, pero también por inversiones estatales en grandes infraestructuras de comunicación, la atracción de fuertes sumas de inversión exterior, junto a un proceso de especulación inmobiliaria y financiera que encontró el apoyo del gobierno regional y buena parte de los gobiernos locales, beneficiarios indirectos de ese dinamismo y poco partidarios de cualquier tipo de regulación.

Ese proceso generó también elevados costes en forma de consumo masivo de suelo, aumento de la movilidad forzada, altos precios de la vivienda, declive de las áreas industriales o creciente segmentación socioespacial, que tuvo como mejor exponente la multiplicación de nuevos servidores vinculados a empleos precarios y mal pagados en la construcción y los servicios al consumo, con destacada presencia de la población inmigrante, que se localizaron sobre todo en las periferias metropolitanas del sur y del este, pero también en algunos enclaves del centro de la ciudad de Madrid. El agotamiento del modelo cargó también buena parte de sus costes sobre estos segmentos sociolaborales y territoriales, aunque está por hacer aún la geografía de la crisis y el análisis de su desigual impacto en la aglomeración.

Pero este tipo de tendencias, que resultan bastante comunes a numerosas regiones metropolitanas europeas más allá de las herencias específicas de cada caso, definen mejor sus perfiles cuando su análisis se complementa con otros realizados desde perspectivas nuevas $\mathrm{O}$, al menos, poco conocidas. En ese sentido, el desarrollo en Madrid de la Ilamada economía del conocimiento, atributo que parece inherente a las ciudades globales, permite añadir a lo anterior dimensiones apenas exploradas. Así, por ejemplo, permite comprobar que las actividades más intensivas en el uso del conocimiento representan ya casi uno de cada tres empleos, si bien distan mucho de ser ajenas al impacto de una crisis como la actual. También que los contratos firmados desde 2005 para ocupaciones de alta cualificación solo representan un $18 \%$ del total y bastantes de ellos adolecen de una elevada precariedad relacionada con su temporalidad, por lo que la aproximación a esta temática desde ambas perspectivas ofrece resultados no coincidentes.

Por último, pese a tratarse de actividades y profesionales que trabajan de forma mayoritaria con recursos inmateriales y potencialmente tan móviles como la información y el conocimiento, supuestamente libres por tanto de cualquier restricción espacial para su implantación, el territorio dista mucho de ser plano a la hora de que empresas e instituciones tomen ese tipo de decisiones de localización. Eso se hace evidente al constatar la persistencia de comportamientos espaciales guiados por una lógica estricta, en la que factores económicos de rentabilidad, junto a otros menos tangibles como el valor simbólico de ciertas áreas centrales o suburbanas de alta calidad, las dinámicas de proximidad y las externalidades que se derivan, la simple inercia o unas políticas locales de promoción activas e innovadoras en algunas ciudades concretas mantienen -e incluso refuerzan- su capacidad para explicar los mapas resultantes.

Pese al riesgo de banalización que siempre acompaña a los términos que ponen de moda los discursos oficiales, la economía del conocimiento ofrece una perspectiva transversal de las economías metropolitanas y abre líneas de investigación a explorar, en la búsqueda de una mejor comprensión de la nueva geografía metropolitana emergente y de las nuevas formas de desigualdad resultantes, base para reflexionar sobre las políticas de promoción y ordenación más adecuadas en cada caso. 


\section{Referencias bibliográficas}

BATHELT, H.; MALMBERG A. y MASKELL, P. Clusters and knowledge: Local buzz, global pipelines and the process of knowledge creation. Progress in Human Geography, 2004, Vol. 28, No 1 , p. 31-56.

BORSDORF, A. La transformación urbanarural en Europa ¿Hacia una unificación espacial en post-suburbia? En: DE MATTOS, C.; FIGUEROA, O.; ORELLANA, A. \& YÁÑEZ, G. (Editores). Gobernanza, competitividad y redes. La gestión de las ciudades en el siglo XXI. Santiago de Chile: Colección Rideal / Eure Libros, Pontificia Universidad Católica de Chile, 2005, p. 21-30.

BURNS, M.C.; ROCA, J.; MOIX, M. y ULIED, M. El sistema metropolitano de la macrorregión de Madrid. Urban, 2009, № 14, p. 72-79.

BURRIEL, E. Subversion of land-use plans and the housing Hubble in Spain. Urban Research \& Practice, Vol. 4, No 3, p. 232-249.

CAPEL, H. Las pequeñas ciudades en la urbanización generalizada y ante la crisis global". Investigaciones Geográficas, 2009, $\mathrm{N}^{\circ} 70$, p. 7-32.

CASTILLO, J. El trabajo recobrado. Una evaluación del trabajo realmente existente en España. Buenos Aires-Madrid: Miño y Dávila, 2005.

COOKE, P. \& LAZZERETTI, L. (Editores). Creative cities, cultural clusters and local economic development. Cheltenham: Edward Elgar, 2008.

DE MATTOS, C. Globalización y metamorfosis metropolitana en América Latina. De la ciudad a lo urbano generalizado. Revista de Geografía Norte Grande, 2010, № 47, p. 81-104.

DE SANTIAGO, E. Madrid ciudad única (II). La explosión urbana en la región madrileña y sus efectos colaterales. Urban, 2008, No 13, p. 138-164.
DEMATTEIS, G. Suburbanización y periurbanización. Ciudades anglosajonas y ciudades Latinas. En: MONCLÚS, F.J. (Ed.). La ciudad dispersa. Suburbanización y nuevas periferias. Barcelona: Centre de Cultura Contemporánia, 1998, p. 17-33.

ERGAZAKIS, K.; Metaxiotis, K. \& Psarras, J. Towards knowledge cities: conceptual analysis and success stories. Journal of Knowledge Management, 2004, Vol. 10, $\mathrm{N}^{\circ}$ 5, p. 65-78.

FLORIDA, R. The rise of the creative class and how it's transforming work, leisure, community and everyday life. Nueva York: Basic Books, 2002.

FUNDACIÓN SINDICAL DE ESTUDIOS. Repercusiones sociales de la crisis en Madrid. Madrid: Fundación Sindical de Estudios de Comisiones Obreras, 2010.

GALLO, M.T.; GARRIDO, R. y VIVAR, M. Cambios territoriales en la Comunidad de Madrid: policentrismo y dispersión. EURE, 2010, Vol. 36, No 107 , p. 5-26.

GARCÍA, J.C. Los desplazamientos al trabajo en la Comunidad de Madrid. Madrid: Ediciones GPS, 2008.

GLAESER, E.L.; KOLKO, J. \& SAIZ, A. Consumer city. Journal of Economic Geography, 2001, No 1, p. 27-50.

GORZ, A. Métamorphoses du travail. Quête du sens. Critique de la raison économique. París: Editions Galilée, 1991.

HALBERT, L. Les métropoles, moteurs de la dématérialisation du Systeme productif Urbain français : une lecture sectorielle et fonctionelle (1982-1999). Bulletin de I'Association des Géographes Françaises, 2005, Vol. 82, No 3, p. 277-299.

HARVEY, D. Espacios del capital. Hacia una geografía crítica. Madrid, Akal, 2007. 
HIDALGO, R. y ZUNINO, H.M. La urbanización de las áreas periféricas en Santiago y Valparaíso: el papel de las relaciones de poder en el dibujo de la geografía sociorresidencial. EURE, 2011, Vol. 37, No 111, p. 79-105.

JANOSCHKA, M. Geografías urbanas en la era del neoliberalismo. Una conceptualización de la resistencia local a través de la participación y la ciudadanía urbana. Investigaciones Geográficas, 2011, № 76, p. 118-132.

KRÄTKE, S. Metropolisation of the european economic territory as a consequence of increasing specialisation of urban agglomerations in the knowledge economy. European Planning Studies, 2007, Vol. 15, No 1, p. 1-27.

LEAL, J. y DOMínGUEZ, M. Pasado y futuro del parque de viviendas en Madrid: despejando incertidumbres. Urban, 2009, $\mathrm{N}^{\circ}$ 14 , p. $80-90$.

LÓPEZ, I. y RODRÍGUEZ, E. Fin de ciclo. Financiarización, territorio y sociedad de propietarios en la onda larga del capitalismo hispano (1959-2010). Madrid: Traficantes de Sueños, 2010.

MARTIN, R. \& SIMMIE, J. Path dependence and local innovation systems in city-regions. Innovation Management Policy\&Practice, 2008, № 10, p. 183-196.

MÉNDEZ, R. El territorio de las nuevas economías metropolitanas. EURE, 2007, Vol. $33, N^{\circ} 100$, p. 51-67.

MÉNDEZ, R. Inmigración y mercados de trabajo urbanos: tendencias recientes en la región metropolitana de Madrid. Scripta Nova, 2008, Vol. XII, № 257. Disponible en Internet: http://www.geocritica.com

MÉNDEZ, R. y RODRÍGUEZ, J. Transformaciones productivas y nuevas formas urbanas: difusión de las actividades económicas en la región metropolitana funcional de Madrid. Anales de Geografía de la Universidad Complutense, 2007, Vol. 27, № 2, p. 105134.
OBSERVATORIO DE LA INMIGRACIÓN. Informe demográfico de la población extranjera en la Comunidad de Madrid. Madrid: Comunidad de Madrid, 2010.

OBSERVATORIO METROPOLITANO. Manifiesto por Madrid. Crítica y crisis del modelo metropolitano. Madrid: Traficantes de Sueños, 2009.

OCDE. OECD Territorial Reviews. Madrid, Spain. París: OECD, 2007.

PECK, J. Struggling with the creative class. International Journal of Urban and Regional Research, 2005, Vol. 29, № 4, p. 740-770.

ROMER, P. Human capital and growth: theory and evidence. NBER Working Paper, $\mathrm{N}^{\circ}$ W3173, 1989.

SÁNCHEZ, S.; CALATRAVA, A. y MELERO. Las funciones comando de Madrid en la economía global: una aproximación a través del proceso de atracción del capital extranjero. EURE, 2008, Vol. 34, № 101, p. 25-44.

SCOTT, A.J. Global city-regions: trends, theory, policy. Oxford: Oxford University Press, 2003.

SCOTT, A. J. Creative cities: conceptual issues and policy questions. Journal of Urban Affairs, 2006, Vol. 28, № 1, p. 1-17.

SCOTT, A.J. Human capital resources and requirements across the metropolitan hierarchy of the USA". Journal of Economic Geography, 2009, No 9, p. 207-226.

SIMMIE, J. \& STRAMBACH, S. The contribution of KIBS to innovation in cities: an evolutionary and institutional perspective. Journal of Knowledge Management, 2006, Vol. 10, № 5, p. 26-40.

SOJA, E. Postmetropolis: Critical studies of cities and regions. Malden: Blackwell, 2000. 
STEHR, N. Knowledge and economic conduct. The social foundations of the modern economy. Toronto: University of Toronto Press, 2002.

TAYLOR, P.J; NI, P.; DERUDDER, B.; HOYLER, M.; HUANG, J. \& WITLOX, F. (Editores). Global urban analysis. A survey of cities in globalization. Londres-Washington D.C.: Earthscan, 2011.
TRULLÉN, J.; LLADÓS, J. y BOIX, R. EConomía del conocimiento, ciudad y competitividad. Investigaciones Regionales, 2002, No 1, p.139-161.

WINDEN, W. VAN; BERG, L. VAN DEN \& POL, P. European cities in the knowledge economy: towards a tipology. Urban Studies, 2007, Vol. 44, No. 3, p. 525-549. 
